Case Report

\title{
A Case of Posttransfusion Purpura with Severe Refractory Thrombocytopenia but No Cutaneous Manifestations
}

\author{
Jagjit Singh Bhamra $\mathbb{D}^{1},{ }^{1}$ Per Ole Iversen, ${ }^{2}$ Thomas Kjenner Titze, ${ }^{1}$ \\ Geir Erland Tjønnfjord ${ }^{D}{ }^{3}$ and Çiğdem Akalın Akkök ${ }^{1}{ }^{1}$ \\ ${ }^{1}$ Department of Immunology and Transfusion Medicine, Oslo University Hospital, Oslo, Norway \\ ${ }^{2}$ Department of Nutrition, Institute of Basic Medical Sciences, University of Oslo, Oslo, Norway \\ ${ }^{3}$ Department of Haematology, Oslo University Hospital, Oslo, Norway
}

Correspondence should be addressed to Çiğdem Akalın Akkök; uxciak@ous-hf.no

Received 22 June 2018; Accepted 8 October 2018; Published 29 October 2018

Academic Editor: Kostas Konstantopoulos

Copyright (c) 2018 Jagjit Singh Bhamra et al. This is an open access article distributed under the Creative Commons Attribution License, which permits unrestricted use, distribution, and reproduction in any medium, provided the original work is properly cited.

Posttransfusion purpura is a serious adverse effect of transfusion due to HPA-antibodies. A young female was diagnosed with acute leukaemia, and treatment commenced. Severe thrombocytopenia ensued. No platelet increment was achieved despite transfusions with buffy coat, HLA-compatible, and HPA-1a negative platelets. The workup indicated the presence of anti-HPA-1a. When the diagnosis of posttransfusion purpura was sufficiently substantiated, she had experienced a fatal intracerebral haemorrhage.

\section{Introduction}

Posttransfusion purpura (PTP) is a rare transfusion reaction. In the British haemovigilance scheme, 56 PTP cases were reported in the period 1996-2015 [1]. PTP can occur following alloimmunisation to human platelet antigens (HPA), most commonly after pregnancies and/or blood transfusions. The antibody-mediated destruction of transfused platelets and the patients' own platelets can precipitate fatal bleedings. The preferred treatment is intravenous immunoglobulin (IVIg) [2].

1.1. Case Presentation. A young primipara was diagnosed with acute myeloid leukaemia (AML). She had haemoglobin (Hb) $9.9 \mathrm{~g} / \mathrm{dL}(11.7-15.3)$, leukocytes $32.3 \times 10^{9} / \mathrm{L}(3.5-11)$, platelets $119 \times 10^{9} / \mathrm{L}(145-390)$, and $\mathrm{D}$-dimer $1.3 \mathrm{mg} / \mathrm{L}$ (0.0-0.4). INR, APTT, and other blood values were normal. Induction chemotherapy started (Figure 1). On day 4, she received two units of leukoreduced packed red blood cells (PRBC) when $\mathrm{Hb}$ was $6.9 \mathrm{~g} / \mathrm{dL}$. On day 7, the induction chemotherapy was completed and antibiotics were initiated because of a lesion on her hand. The following night, she fainted and had haematochezia. $\mathrm{Hb}$ was $8.1 \mathrm{~g} / \mathrm{dL}$, and platelets were $12 \times 10^{9} / \mathrm{L}$; hence, one PRBC and one buffy coat platelet concentrate (BCPC) were given. Four more BCPCs were given on days 8-9 without any platelet increment. Luminex single-antigen assay revealed weak class I HLA-antibodies. Three HLA-compatible platelet concentrates (HCPCs) were transfused on days 10-12 but with no platelet increment. On day 13, one unit of HLA-compatible HPA-1bb platelet concentrate was transfused as HPAantibodies were suspected, still without any platelet increment. Another HCPC was transfused on day 14 when she fell hitting her head.

On day 15, she presented with an acute stroke. Prothrombin complex concentrate, recombinant factor VIIa, four HCPCs, two PRBCs, and intravenous tranexamic acid were administered. A CT scan revealed an intracerebral haemorrhage $(\mathrm{ICH})$ that was evacuated, but perioperative haemostasis was not achieved. She died of cerebral herniation.

A flow cytometric investigation on day 13 revealed increased reactivity to platelets from six HPA-1a positive 


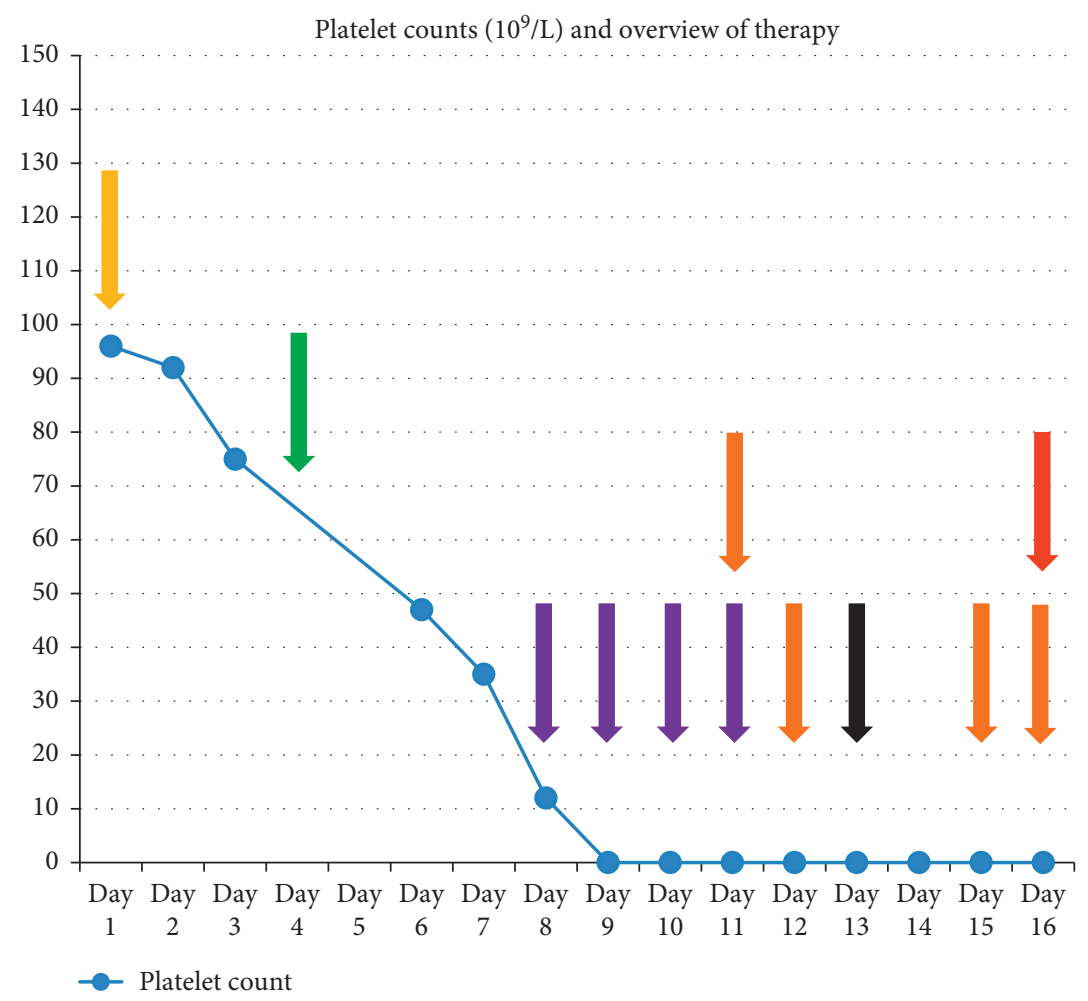

Figure 1: Platelet counts $\left(10^{9} / \mathrm{L}\right)$ in our patient with AML, in relation to induction chemotherapy and all transfusions. Purple arrow indicates BCPC (buffy coat platelet concentrate): Day 8: BCPC $\times 2$. Day 9: BCPC $\times 2$. Day 10: BCPC $\times 1$. Day 11: BCPC $\times 1$. Orange arrow indicates HCPC (HLA-compatible platelet concentrates): Day 11: HCPC $\times 2$. Day 12: HCPC $\times 1$. Day 15: HCPC $\times 1$. Day 16: HCPC $\times 4$. Green arrow indicates PRBC (packed red blood cells): Day 4: PRBC $\times 2$. Yellow arrow indicates Day 1: Start induction chemotherapy. Black arrow indicates Day 13: HPA-1bb platelet concentrate $\mathrm{x}$ 1. Red arrow indicates Day 16: Death.

donors and to lymphocytes from two of these donors. A crossmatch between the patient's plasma and platelets from an HPA-1bb donor was negative. These findings indicated the presence of anti-HPA-1a and a probable PTP.

\section{Discussion}

Our patient was transfused with two PRBCs, which probably triggered an anamnestic response by boosting anti-HPA-1a titres after a likely alloimmunisation during pregnancy. Four days after those transfusions, her platelet count was $<10 \times$ $10^{9} / \mathrm{L}$, and it never increased despite repeated transfusions. The workup was consistent with the presence of anti-HPA1a. Postmortem, she was genotyped to HPA-1bb and HLADRB3*0101 positive, further supporting a PTP diagnosis.

Anti-HPAs can cause PTP and foetal neonatal alloimmune thrombocytopenia. Approximately 2\% of Caucasians are positive for HPA-1bb [3]. Anti-HPA-1a made by them is the culprit antibody in $80-90 \%$ of PTP cases $[3,4]$. PTP was first described by Shulman and coworkers [5]. The reported incidence is $1: 50000-1: 100000$ [6], though PTP is likely underdiagnosed. The typical patient is a middle-aged, HPAlbb female, who has been alloimmunised to HPA-1a in pregnancies and/or by blood transfusions. Male patients have been described [4]. Renewed exposure to the same antigen provokes an anamnestic response boosting alloantibody production. Other HPA-antibodies can also cause PTP $[1,3,6]$.

Severe thrombocytopenia occurs 2-14 days [7] after the transfusion of a platelet-containing product (e.g., PRBCs, whole blood, platelet concentrates, and fresh plasma) with the foreign antigen $[4,6,8]$. Besides the transfused antigenpositive platelets, the recipient's antigen-negative platelets are also destroyed. Several mechanisms have been proposed to explain why: (1) Platelet antigen-positive blood transfusion triggers production of autoantibodies [9], (2) Transfused HPA-1a antigens are adsorbed onto the patient's own platelets [3], and (3) A cross-reaction between antiHPA-1a and the patient's own platelets [10].

Symptoms include mucosal bleedings, haematomas, melena, haematuria, epistaxis, abnormal postoperative bleedings, and ICH. Not all have cutaneous manifestations [11]. Coagulation screens and bone marrow biopsies are usually normal [3], though obviously this would not be the case in our patient with AML. Untreated PTP lasts 7-28 days but can persist longer [3].

A thorough investigation can cause a diagnostic delay, which may warrant starting treatment before the workup is completed. Treatment with steroids and exchange transfusions [4] takes days and weeks before the thrombocytopenia resolves. About $80 \%$ of cases respond with a platelet increment within 48-72 hours following the administration of IVIg of $1-2 \mathrm{~g} / \mathrm{kg}$ for two to five days or $500 \mathrm{mg} / \mathrm{kg}$ for five days [1-3]. The indication for platelet transfusions is 
controversial; however, the potential for preventing fatal bleedings may imply a liberal transfusion strategy.

Heparin-induced thrombocytopenia (HIT), disseminated intravascular coagulation (DIC), drug-induced thrombocytopenia (DIT), septicaemia, underlying haematological diseases, thrombotic microangiopathies, immune thrombocytopenia (ITP), and splenomegaly must be excluded [3]. Extraordinarily, passively transfused HPAantibodies from a blood donor may cause a PTP-like condition in the recipient with shorter times for onset and spontaneous resolution [3]. A review of the medications our patient received excluded HIT and DIT. No findings were consistent with DIC, septicaemia, thrombotic microangiopathies, ITP, or splenomegaly. None of the abovementioned conditions have a temporal relationship with a preceding transfusion, unlike PTP.

HLA-antibodies can be present in PTP patients [3], necessitating further analyses to identify the culprit alloantibody. Monoclonal antibody-specific immobilisation of platelet antigen assay (MAIPA) or Luminex-based assay is used to resolve the presence and specificity of the HPAantibody. HLA-DRB3*0101 typing should be performed as this tissue type is assumed to play an HPA-1a antigenpresenting role [12] and HLA-DRB3*0101 individuals are prone to producing anti-HPA-1a. A drawback of our investigation was that MAIPA was not performed due to lack of a suitable sample.

\section{Conclusion}

Not all PTP patients have purpura justifying the name of the condition, and the lack of this may lead to diagnostic and therapeutic delays. However, refractoriness to all kinds of platelet transfusions is the typical finding. We, therefore, suggest this condition should be called posttransfusion refractory thrombocytopenia (PTRT).

\section{Conflicts of Interest}

The authors declare that there are no conflicts of interest regarding the publication of this article.

\section{References}

[1] P. H. B. Bolton-Maggs and D. Poles, On Behalf of the Serious Hazards of Transfusion (SHOT) Steering Group: The 2015 Annual Shot Report, SHOT Office, Manchester Blood Centre, Manchester, UK, 2016.

[2] L. M. Williamson, D. Stainsby, H. Jones et al., "The impact of universal leukodepletion of the blood supply on hemovigilance reports of posttransfusion purpura and transfusionassociated graft-versus-host disease," Transfusion (Paris), vol. 47, no. 8, pp. 1455-1467, 2007.

[3] M. F. Murphy, Posttransfusion Purpura; Practical Transfusion Medicine, John Wiley \& Sons, Ltd, Hoboken, NJ, USA, 2013.

[4] C. Mueller-Eckhardt, "Post-transfusion purpura," British Journal of Haematology, vol. 64, no. 3, pp. 419-424, 1986.

[5] N. R. Shulman, R. H. Aster, A. Leitner et al., "Immunoreactions involving platelets. V. Post-transfusion purpura due to a complement-fixing antibody against a genetically controlled platelet antigen. A proposed mechanism for thrombocytopenia and its relevance in autoimmunity," Journal of Clinical Investigation, vol. 40, no. 9, pp. 1597-1620, 1961.

[6] O. P. Arewa, S. Nahirniak, and G. Clarke, "Anti-HPA-1b mediated posttransfusion purpura: a case report," Case Reports in Medicine, vol. 2013, Article ID 568364, 3 pages, 2013.

[7] M. M. Cushing, "Transfusion medicine and hemostasis," in Clinical and Laboratory Aspects, pp. 433-434, Elsevier, Amsterdam, Netherlands, 2013.

[8] P. L. Cimo and R. H. Aster, "Post-transfusion purpura: successful treatment by exchange transfusion," New England Journal of Medicine, vol. 287, no. 6, pp. 290-292, 1972.

[9] R. B. Stricker, B. H. Lewis, L. Corash et al., "Posttransfusion purpura associated with an autoantibody directed against a previously undefined platelet antigen," Blood, vol. 69 , no. 5 , pp. 1458-1463, 1987.

[10] N. R. Shulman and J. V. Jordan, "Platelet immunology," in Hemostasis and Thrombosis, R. W. Colman JH, V. J. Marder, and E. W. Salpnan, Eds., p. 274, Lippincott Williams \&Wilkins, Philadelphia, PA, USA, 1982.

[11] P. H. B. Bolton-Maggs, D. Poles, A. Watt, and D. Thomas, On Behalf of the Serious Hazards of Transfusion (SHOT) Steering, Group: The 2013 Annual SHOT Report, SHOT Office, Manchester Blood Centre, Manchester, UK, 2014.

[12] D. L'Abbe, L. Tremblay, M. Filion et al., "Alloimmunization to platelet antigen HPA-1a (PIA1) is strongly associated with both HLA-DRB $3 * 0101$ and HLA-DQB $1 * 0201$," Human Immunology, vol. 34, no. 2, pp. 107-114, 1992. 


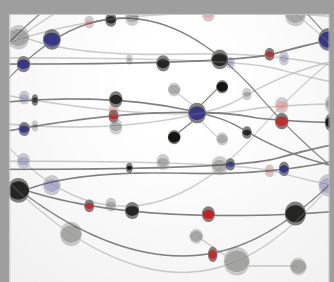

The Scientific World Journal
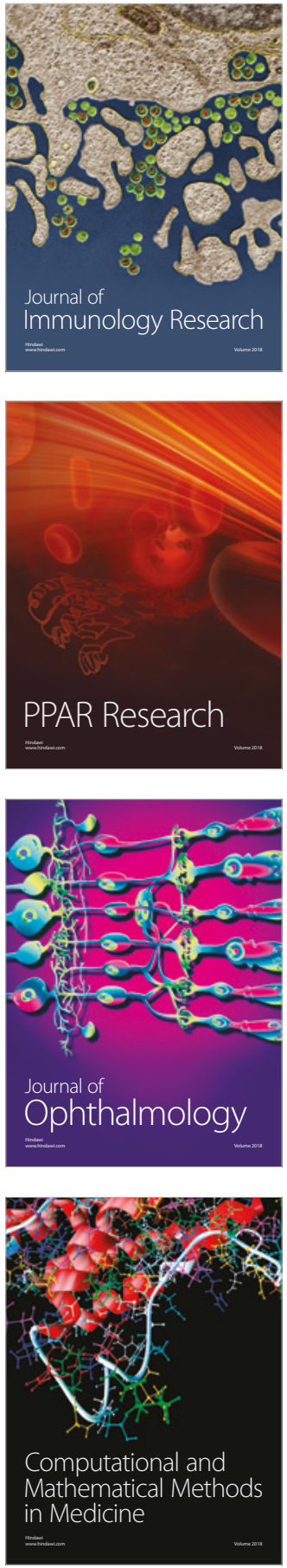

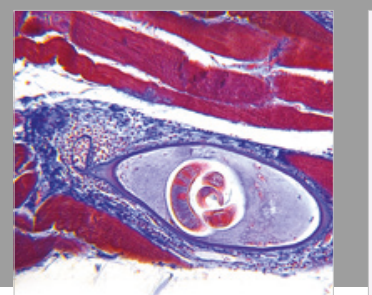

Gastroenterology Research and Practice

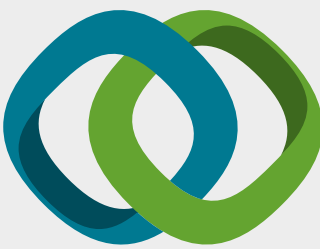

\section{Hindawi}

Submit your manuscripts at

www.hindawi.com
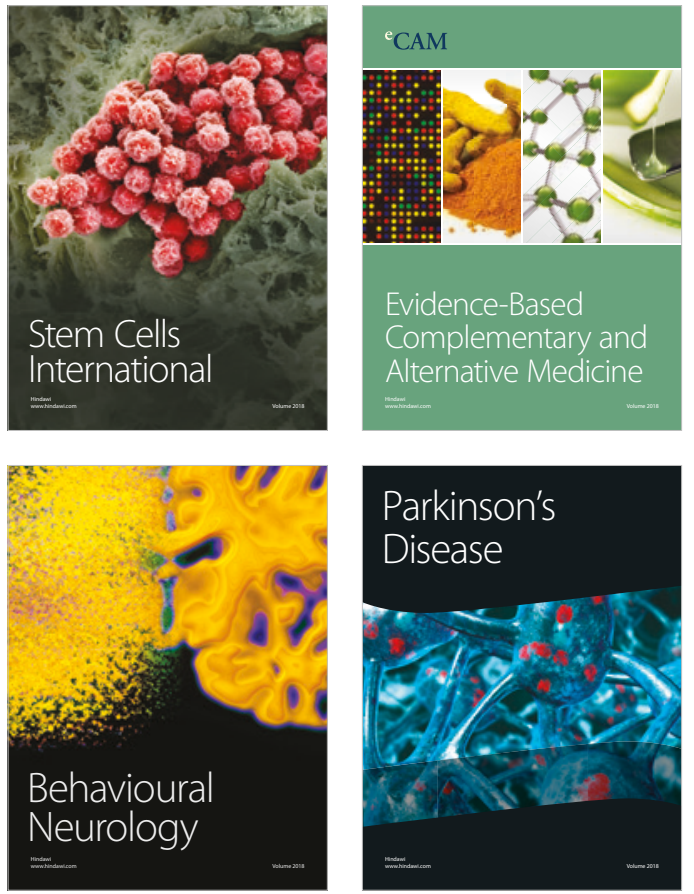

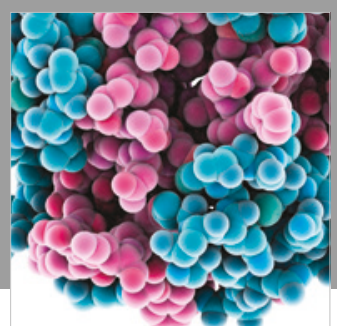

ournal of

Diabetes Research

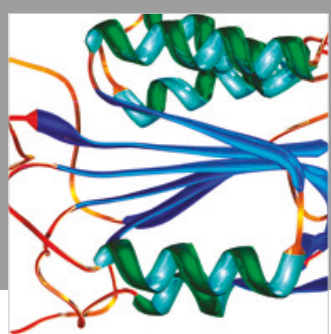

Disease Markers
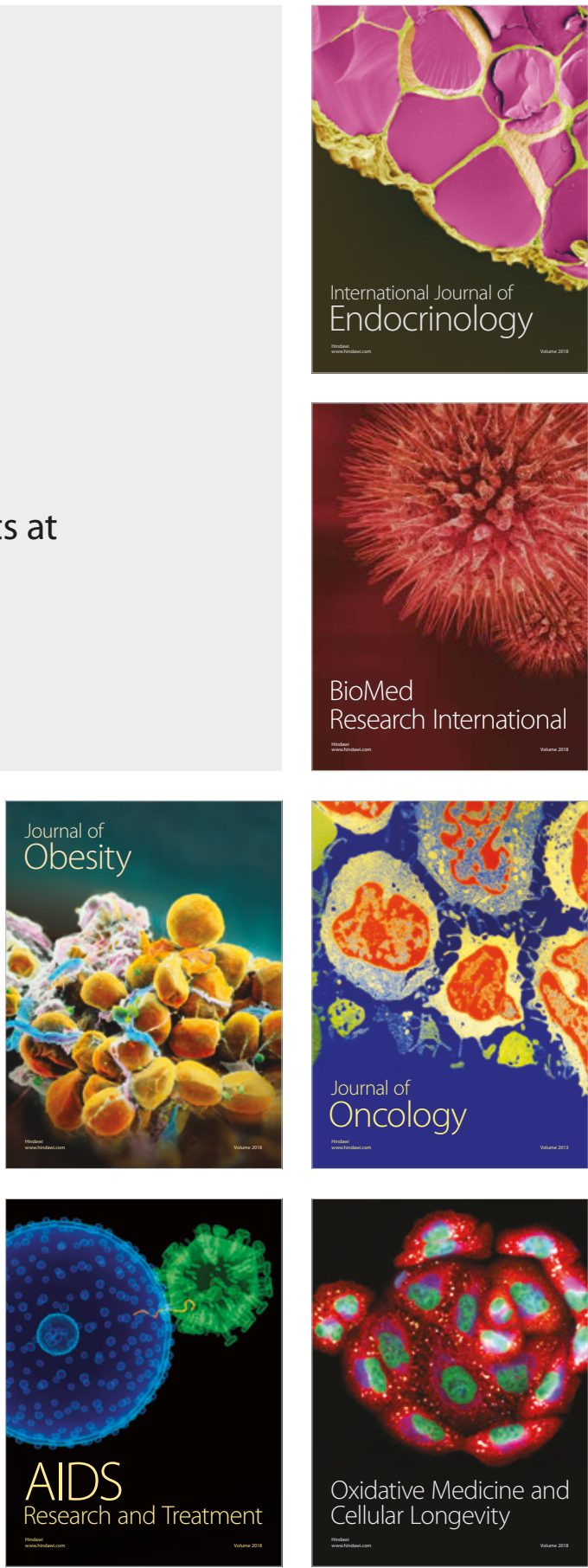